\title{
Extinction and asymptotic behavior of solutions for nonlinear parabolic equations with variable exponent of nonlinearity
}

\section{Yanchao Gao* and Wenjie Gao}

"Correspondence:

ychaogao@163.com

School of Mathematics, Jilin

University, Changchun, 130012, P.R.

China

\begin{abstract}
The aim of this paper is to study the existence and extinction of weak solutions of the initial and boundary value problem for $u_{t}=\operatorname{div}\left(\left(|u|^{\sigma(x, t)}+d_{0}\right)|\nabla u|^{p(x, t)-2} \nabla u\right)+f(x, t, u)$. First, the authors apply the method of parabolic regularization and Galerkin's method to prove the existence of solutions to the problem mentioned and then obtain the comparison principle by arguing by contradiction. Furthermore, the authors prove that the solution vanishes in finite time and approaches 0 in $L^{2}(\Omega)$ norm as $t \rightarrow \infty$.
\end{abstract}

Keywords: nonlinear parabolic equation; nonstandard growth condition; extinction; $p(x, t)$-Laplace operator

\section{Introduction}

Let $\Omega \subset \mathbb{R}^{N}(N \geq 1)$ be a bounded simply connected domain and $0<T<\infty$. Consider the following quasilinear degenerate parabolic problem:

$$
\left\{\begin{array}{l}
u_{t}=\operatorname{div}\left(a(x, t, u)|\nabla u|^{p(x, t)-2} \nabla u\right)+f(x, t, u), \quad(x, t) \in Q_{T}, \\
u(x, t)=0, \quad(x, t) \in \Gamma_{T}, \\
u(x, 0)=u_{0}(x), \quad x \in \Omega
\end{array}\right.
$$

where $Q_{T}=\Omega \times(0, T], \Gamma_{T}$ denotes the lateral boundary of the cylinder $Q_{T}, a(x, t, u)=$ $|u|^{\sigma(x, t)}+d_{0}$ with the assumption that $d_{0}$ is a positive constant and the nonlinear source $f(x, t, u)$ satisfies

$$
f(x, t, u)=b(x, t)-b_{0} u(x, t), \quad u \in(-\infty,+\infty), x \in \Omega, t>0
$$

with $b_{0}>0, b(x, t) \geq 0,(x, t) \in Q_{T}$. It will be assumed throughout the paper that the exponents $p(x, t), \sigma(x, t)$ are continuous in $Q=\overline{Q_{T}}$ with the logarithmic module of continuity:

$$
\begin{aligned}
& 1<p^{-}=\inf _{(x, t) \in Q} p(x, t) \leq p(x, t) \leq p^{+}=\sup _{(x, t) \in Q} p(x, t)<\infty, \\
& 0<\sigma^{-}=\inf _{(x, t) \in Q} \sigma(x, t) \leq \sigma(x, t) \leq \sigma^{+}=\sup _{(x, t) \in Q} \sigma(x, t)<\infty, \\
& \forall z=(x, t) \in Q_{T}, \xi=(y, s) \in Q_{T},|z-\xi|<1, \quad|p(z)-p(\xi)| \leq \omega(|z-\xi|),
\end{aligned}
$$

๑ 2013 Gao and Gao; licensee Springer. This is an Open Access article distributed under the terms of the Creative Commons Attribution License (http://creativecommons.org/licenses/by/2.0), which permits unrestricted use, distribution, and reproduction in any medium, provided the original work is properly cited. 
where

$$
\limsup _{\tau \rightarrow 0^{+}} \omega(\tau) \ln \frac{1}{\tau}=C<+\infty .
$$

Model (1.1) may describe some properties of image restoration in space and time. Especially when the nonlinear source $f(x, u)=b(x, t)-b_{0} u$, the functions $u(x, t), b(x, t)$ represent a recovering image and its observed noisy image, respectively. In the case when $p(x, t)$, $\sigma(x, t)$ are fixed constants, there have been many results about the existence, uniqueness, blowing-up and so on; we refer to the bibliography [1-3]. When $p, \sigma$ are functions with respect to the space variable and time variable, this problem arises from elastic mechanics, electro-rheological fluids dynamics and image processing, etc.; see [4-9].

To the best of our knowledge, there are only a few works about parabolic equations with variable exponents of nonlinearity. In [6], Chen, Levine and Rao obtained the existence and uniqueness of weak solutions with the assumption that the exponent $\sigma(x, t) \equiv 0$, $1<p^{-}<p^{+}<2$. In [10], we applied the method of parabolic regularization and Galerkin's method to prove the existence of weak solutions to problem (1.1) with the assumption that $\sigma(x, t) \equiv$ constant, $f(x, t, u) \equiv f(x, t)$. In this paper, we generalize the results in [10]. Especially, unlike [10], we obtain the existence and uniqueness of weak solution not only in the case when $\sigma(x, t) \in\left(2, \frac{2 p^{+}}{p^{+}-1}\right), p^{+} \geq 2$, but also in the case when $\sigma(x, t) \in(1,2)$, $1<p^{-}<p^{+} \leq 1+\sqrt{2}$. Furthermore, we apply energy estimates and Gronwall's inequality to obtain the extinction of solutions when the exponents $p^{-}$and $p^{+}$belong to different intervals; as we know such results are seldom seen for the problems with variable exponents. At the end of this paper, we prove that the solution approaches 0 in $L^{2}(\Omega)$ norm as $t \rightarrow \infty$ by some techniques in convex analysis.

The outline of this paper is the following. In Section 2, we introduce the function spaces of Orlicz-Sobolev type, give the definition of weak solution to the problem and prove the existence of weak solutions with a method of regularization and the uniqueness of solutions by arguing by contradiction. Section 3 is devoted to the proof of the extinction of the solution obtained in Section 2. In Section 4, we get the long time asymptotic behavior of the solution.

\section{Existence and uniqueness of weak solutions}

We study the existence of weak solutions in this section. Let us introduce the Banach spaces

$$
\begin{aligned}
& \mathbf{L}^{p(x, t)}\left(Q_{T}\right)=\left\{u(x, t) \mid u \text { is measurable in } Q_{T}, A_{p(\cdot)}(u)=\iint_{Q_{T}}|u|^{p(x, t)} d x d t<\infty\right\}, \\
& \|u\|_{p(\cdot)}=\inf \left\{\lambda>0, A_{p(\cdot)}(u / \lambda) \leq 1\right\} ; \\
& \mathbf{V}_{t}(\Omega)=\left\{u\left|u \in L^{2}(\Omega) \cap W_{0}^{1,1}(\Omega),\right| \nabla u \mid \in L^{p(x, t)}(\Omega)\right\}, \\
& \|u\|_{\mathbf{V}_{t}(\Omega)}=\|u\|_{2, \Omega}+\|\nabla u\|_{p(., t) \Omega ;} \\
& \mathbf{W}\left(Q_{T}\right)=\left\{u:[0, T] \mapsto \mathbf{V}_{t}(\Omega)\left|u \in L^{2}\left(Q_{T}\right),\right| \nabla u \mid \in L^{p(x, t)}\left(Q_{T}\right), u=0 \text { on } \Gamma_{T}\right\}, \\
& \|u\|_{\mathbf{W}_{\left(Q_{T}\right)}}=\|u\|_{2, Q_{T}}+\|\nabla u\|_{p(x, t), Q_{T}}
\end{aligned}
$$

and denote by $\mathbf{W}^{\prime}\left(Q_{T}\right)$ the dual of $\mathbf{W}\left(Q_{T}\right)$ with respect to the inner product in $L^{2}\left(Q_{T}\right)$. 
Definition 2.1 A function $u(x, t) \in \mathbf{W}\left(Q_{T}\right) \cap L^{\infty}\left(0, T ; L^{\infty}(\Omega)\right)$ is called a weak solution of problem (1.1) if for every test-function

$$
\xi \in \mathcal{Z} \equiv\left\{\eta(z): \eta \in \mathbf{W}\left(Q_{T}\right) \cap \mathbf{L}^{\infty}\left(0, T ; L^{2}(\Omega)\right), \eta_{t} \in \mathbf{W}^{\prime}\left(Q_{T}\right)\right\},
$$

and every $t_{1}, t_{2} \in[0, T]$, the following identity holds:

$$
\begin{aligned}
& \int_{t_{1}}^{t_{2}} \int_{\Omega}\left[u \xi_{t}-\left(|u|^{\sigma(x, t)}+d_{0}\right)|\nabla u|^{p(x, t)-2} \nabla u \nabla \xi+f(x, t, u) \xi\right] d x d t \\
& \quad=\left.\int_{\Omega} u \xi d x\right|_{t_{1}} ^{t_{2}} .
\end{aligned}
$$

Following the line of the proof of Theorem 2.1 in $[10,11]$, we have the following theorem about the existence of weak solutions.

Theorem 2.1 Let the function $f(x, t, u)$ and the exponents $p(x, t), \sigma(x, t)$ satisfy Conditions (1.2)-(1.5). If the following conditions hold:

$\left(\mathrm{H}_{1}\right) \quad \max \left\{1, \frac{2 N}{N+2}\right\}<p^{-}$,

$$
\frac{\sigma^{+}\left(p^{+}-1\right)}{p^{+}} \leq \sigma^{-}<\sigma^{+}<2 \quad \text { or } \quad 2 \leq \sigma^{-}<\sigma^{+}<\frac{2 p^{+}}{p^{+}-1} \text {; }
$$

$\left(\mathrm{H}_{2}\right) \quad\left\|u_{0}\right\|_{\infty, \Omega}+\int_{0}^{T}\|b(x, t)\|_{\infty, \Omega} d t=K(T)<\infty$,

then problem (1.1) has at least one weak solution $u$ satisfying $\|u\|_{\infty, Q_{T}} \leq\left\|u_{0}\right\|_{\infty, \Omega}$.

The theorems about the uniqueness of weak solutions are as follows.

Theorem 2.2 Suppose that the conditions in Theorem 2.1 are fulfilled and the following condition is satisfied:

$$
\left(\mathrm{H}_{3}\right) \quad \frac{\sigma^{+}\left(p^{+}-1\right)}{p^{+}} \leq \sigma^{-}<\sigma^{+}<2, \quad 1<p^{-}<p^{+} \leq 1+\sqrt{2} .
$$

Then the nonnegative bounded solution of problem (1.1) is unique within the class of all nonnegative bounded weak solutions.

Proof We argue by contradiction. Suppose that $u(x, t)$ and $v(x, t)$ are two nonnegative weak solutions of problem (1.1) and there is a $\delta>0$ such that for some $0<\tau \leq T, w=u-v>\delta$ on the set $\Omega_{\delta}=\Omega \cap\{x: w(x, \tau)>\delta\}$ and $\mu\left(\Omega_{\delta}\right)>0$. Let

$$
F_{\varepsilon}(\xi)= \begin{cases}\frac{1}{1-\alpha} \varepsilon^{\alpha-1}-\frac{1}{1-\alpha} \xi^{\alpha-1} & \text { if } \xi>\varepsilon \\ 0 & \text { if } \xi \leq \varepsilon\end{cases}
$$

where $\delta>2 \varepsilon>0$ and $\alpha=\frac{\sigma^{+}}{2}$. 
By the definition of weak solutions, pick a test-function $\xi=F_{\varepsilon}(w) \in \mathcal{Z}$,

$$
\begin{aligned}
0= & \iint_{Q_{\tau}}\left[w_{t} F_{\varepsilon}(w)+\left(v^{\sigma(x, t)}+d_{0}\right)\left(|\nabla u|^{p(x, t)-2} \nabla u-|\nabla v|^{p(x, t)-2} \nabla v\right) \nabla F_{\varepsilon}(w)\right] d x d t \\
& +\iint_{Q_{\tau}}\left(u^{\sigma(x, t)}-v^{\sigma(x, t)}\right)|\nabla u|^{p(x, t)-2} \nabla u \nabla F_{\varepsilon}(w) d x d t \\
& -\iint_{Q_{\tau}}[f(x, t, u)-f(x, t, v)] F_{\varepsilon}(w) d x d t \\
= & \iint_{Q_{\varepsilon, \tau}} w_{t} F_{\varepsilon}(w) d x d t \\
& +\iint_{Q_{\varepsilon, \tau}}\left(v^{\sigma(x, t)}+d_{0}\right) w^{\alpha-2}\left(|\nabla u|^{p(x, t)-2} \nabla u-|\nabla v|^{p(x, t)-2} \nabla v\right) \nabla w d x d t \\
& +\iint_{Q_{\varepsilon, \tau}}\left(u^{\sigma(x, t)}-v^{\sigma(x, t)}\right) w^{\alpha-2}|\nabla u|^{p(x, t)-2} \nabla u \nabla w d x d t \\
& +\iint_{Q_{\varepsilon, \tau}} w F_{\varepsilon}(w) d x d t:=J_{1}+J_{2}+J_{3}+J_{4},
\end{aligned}
$$

with $Q_{\varepsilon, \tau}=Q_{\tau} \cap\left\{(x, t) \in Q_{\tau}: w>\varepsilon\right\}$.

Now, let $t_{0}=\inf \{t \in(0, \tau]: w>\varepsilon\}$, then we estimate $J_{1}, J_{2}, J_{3}, J_{4}$ as follows:

$$
\begin{aligned}
J_{1} & =\iint_{Q_{\varepsilon, \tau}} w_{t} F_{\varepsilon}(w) d x d t=\int_{\Omega}\left(\int_{0}^{t_{0}} w_{t} F_{\varepsilon}(w) d t+\int_{t_{0}}^{\tau} w_{t} F_{\varepsilon}(w) d t\right) d x \\
& \geq \int_{\Omega} \int_{\varepsilon}^{w(x, \tau)} F_{\varepsilon}(s) d s d x \geq \int_{\Omega_{\varepsilon}} \int_{\varepsilon}^{w(x, \tau)} F_{\varepsilon}(s) d s d x \\
& \geq \int_{\Omega_{\delta}}(w-2 \varepsilon) F_{\varepsilon}(2 \varepsilon) d x \geq(\delta-2 \varepsilon) F_{\varepsilon}(2 \varepsilon) \mu\left(\Omega_{\delta}\right) \\
J_{4} & =\iint_{Q_{\varepsilon, \tau}} w F_{\varepsilon}(w) d x d t \geq 0 .
\end{aligned}
$$

Let us consider first the case $2 \leq p^{-}<p^{+} \leq 1+\sqrt{2}$. By virtue of the first inequality of Lemma 4.4 in [2], we get

$$
\begin{aligned}
J_{2} & =\iint_{Q_{\varepsilon, \tau}}\left(v^{\sigma(x, t)}+d_{0}\right) w^{\alpha-2}\left(|\nabla u|^{p(x, t)-2} \nabla u-|\nabla v|^{p(x, t)-2} \nabla v\right) \nabla w d x d t \\
& \geq \iint_{Q_{\varepsilon, \tau}}\left(v^{\sigma(x, t)}+d_{0}\right) w^{\alpha-2} 2^{-p(x, t)}|\nabla w|^{p(x, t)} d x d t \\
& \geq 2^{-p^{+}} \iint_{Q_{\varepsilon, \tau}}\left(v^{\sigma(x, t)}+d_{0}\right) w^{\alpha-2}|\nabla w|^{p(x, t)} d x d t \geq 0 .
\end{aligned}
$$

According to the condition $\left(\mathrm{H}_{3}\right)$, we have

$$
\begin{aligned}
\frac{2 p^{+}-4}{p^{+}-1} \leq \frac{\sigma^{+}\left(p^{+}-1\right)}{p^{+}}<\sigma^{+} & \Rightarrow 0<\frac{\left(4-\sigma^{+}\right)\left(p^{+}-1\right)}{2 p^{+}}<1 \\
& \Rightarrow \quad \frac{\left(4-\sigma^{+}\right)\left(p^{+}-1\right)}{2 p^{+}} \frac{p(x, t)}{p(x, t)-1} \geq 2-\frac{\sigma^{+}}{2}=2-\alpha
\end{aligned}
$$


and then applying Young's inequality, we may estimate the integrand of $J_{3}$ in the following way:

$$
\begin{aligned}
& \left.\left|\left(u^{\sigma(x, t)}-v^{\sigma(x, t)}\right) w^{\alpha-2}\right| \nabla u\right|^{p(x, t)-2} \nabla u \nabla w \mid \\
& \quad=\left.\left|\sigma(x, t) w \int_{0}^{1}(\theta u+(1-\theta) v)^{\sigma(x, t)-1} d \theta w^{\alpha-2}\right| \nabla u\right|^{p(x, t)-2} \nabla u \nabla w \mid \\
& \quad \leq \frac{C}{w^{2-\alpha}}\left[\frac{v^{\sigma(x, t)}+d_{0}}{C}|\nabla w|^{p(x, t)}+C_{1}\left(\sigma^{ \pm}, d_{0}, K(T), p^{ \pm}\right)|w|^{\frac{\left(4-\sigma^{+}\right)\left(p^{+}-1\right)}{2 p^{+}} \frac{p(x, t)}{p(x, t)-1}}|\nabla u|^{p(x, t)}\right] \\
& \quad=\frac{\left(v^{\sigma(x, t)}+d_{0}\right)}{2^{p^{+}+1} w^{2-\alpha}}|\nabla w|^{p(x, t)}+C_{1}\left(\sigma^{ \pm}, d_{0}, K(T), p^{ \pm}\right)|w|^{\frac{\left(4-\sigma^{+}\right)\left(p^{+}-1\right)}{2 p^{+}} \frac{p(x, t)}{p(x, t)-1}+\alpha-2}|\nabla u|^{p(x, t)} \\
& \quad \leq \frac{\left(v^{\sigma(x, t)}+d_{0}\right)}{2^{p^{+}+1} w^{2-\alpha}}|\nabla w|^{p(x, t)}+C_{1}\left(\sigma, d_{0}, K(T), p^{ \pm}\right)|\nabla u|^{p(x, t)} .
\end{aligned}
$$

Substituting (2.5) into $J_{3}$, we get

$$
J_{3} \leq \frac{1}{2} J_{2}+C \iint_{Q_{\varepsilon, \tau}}|\nabla u|^{p(x, t)} d x d t .
$$

Secondly, we consider the case $1<p^{-}<2,1<p^{+} \leq 1+\sqrt{2}$. According to the second inequality of Lemma 4.4 in [2], it is easily seen that the following inequalities hold:

$$
\begin{aligned}
J_{2} & =\iint_{Q_{\varepsilon, \tau}}\left(v^{\sigma(x, t)}+d_{0}\right) w^{\alpha-2}\left(|\nabla u|^{p(x, t)-2} \nabla u-|\nabla v|^{p(x, t)-2} \nabla v\right) \nabla w d x d t \\
& \geq\left(p^{-}-1\right) \iint_{Q_{\varepsilon, \tau}}\left(v^{\sigma(x, t)}+d_{0}\right) w^{\alpha-2}(|\nabla u|+|\nabla v|)^{p(x, t)-2}|\nabla w|^{2} d x d t \geq 0 .
\end{aligned}
$$

Using Young's inequality, we may evaluate integrand of $J_{3}$ as follows:

$$
\begin{aligned}
\left.\left|\left(u^{\sigma(x, t)}-v^{\sigma(x, t)}\right) w^{\alpha-2}\right| \nabla u\right|^{p(x, t)-2} \nabla u \nabla w \mid \\
=\left.\left|\sigma(x, t) w \int_{0}^{1}(\theta u+(1-\theta) v)^{\sigma(x, t)-1} d \theta w^{\alpha-2}\right| \nabla u\right|^{p(x, t)-2} \nabla u \nabla w \mid \\
\leq \frac{\left(v^{\sigma(x, t)}+d_{0}\right)\left(p^{-}-1\right)}{2 w^{2-\alpha}}(|\nabla u|+|\nabla v|)^{p(x, t)-2}|\nabla w|^{2} \\
\quad+C_{1}\left(\sigma^{ \pm}, d_{0}, K(T), p^{ \pm}\right)|w|^{\frac{4-\sigma^{+}}{2}-2+\alpha}(|\nabla u|+|\nabla v|)^{p(x, t)} \\
\leq \\
\leq \frac{\left(v^{\sigma}+d_{0}\right)\left(p^{-}-1\right)}{2 w^{2-\alpha}}(|\nabla u|+|\nabla v|)^{p(x, t)-2}|\nabla w|^{2}+C_{1}(|\nabla u|+|\nabla v|)^{p(x, t)} .
\end{aligned}
$$

Plugging (2.8) into $J_{3}$, we get

$$
J_{3} \leq \frac{1}{2} J_{2}+C \iint_{Q_{\varepsilon, \tau}}(|\nabla u|+|\nabla v|)^{p(x, t)} d x d t .
$$

Plugging the above estimates (2.3), (2.4), (2.6) and (2.3), (2.7), (2.9) into (2.2) and dropping the nonnegative terms, we arrive at the inequality

$$
(\delta-2 \varepsilon)\left(1-2^{\alpha-1}\right) \varepsilon^{\alpha-1} \mu\left(\Omega_{\delta}\right) \leq \widetilde{C},
$$

with a constant $\widetilde{C}$ independent of $\varepsilon$. 
Noticing that $\lim _{\varepsilon \rightarrow 0}(\delta-2 \varepsilon)\left(1-2^{\alpha-1}\right) \varepsilon^{\alpha-1} \mu\left(\Omega_{\delta}\right)=+\infty$, we obtain a contradiction. This means $\mu\left(\Omega_{\delta}\right)=0$ and $w \leq 0$, a.e. in $Q_{\tau}$.

In the case when $\sigma^{-}>2$, following the lines of the proof of Theorem 2.2, we have the following theorem.

Theorem 2.3 Suppose that the conditions in Theorem 2.1 are fulfilled and the following condition is satisfied:

$$
\left(\mathrm{H}_{4}\right) \quad 2<\sigma^{-}<\sigma^{+}<\frac{2 p^{+}}{p^{+}-1}, \quad p^{+} \geq 2
$$

Then the nonnegative solution of problem (1.1) is unique within the class of all nonnegative weak solutions.

\section{Localization of weak solutions}

In this section, we study the localization of the weak solution to problem (1.1). Namely, we study the extinction of the solution. We discuss the extinction of weak solutions in the case of $\frac{2 N}{N+2} \leq p^{-}<p^{+}<2$ and $1<p^{-}<\frac{2 N}{N+2}, 1<p^{+}<\frac{N p^{-}}{N-p^{-}}$, respectively. Our main results are the following.

Theorem 3.1 Suppose that $b(x, t) \equiv 0, \frac{2 N}{N+2} \leq p^{-}<p^{+}<2$, then any bounded nonnegative solution of problem (1.1) vanishes in finite time for any nonnegative initial data $0 \neq u_{0} \in$ $L^{\infty}(\Omega) \cap W^{1, p(x)}(\Omega)$ and satisfies the following estimate:

$$
\begin{aligned}
& \|u\|_{2}^{2} \leq\left[\left\|u_{0}\right\|_{2}^{\frac{2-p^{+}}{2}}+\frac{C_{1}}{b_{0}} e^{\frac{p^{+}-2}{2} b_{0} t}-\frac{C_{1}}{b_{0}}\right]^{\frac{2}{2-p^{+}}}, \quad 0<t<T_{1}^{*} ; \\
& \|u\|_{2}=0, \quad t \in\left[T_{1}^{*},+\infty\right),
\end{aligned}
$$

where $T_{1}^{*}=\frac{2}{b_{0}\left(2-p^{+}\right)} \ln \left(1+\frac{b_{0}}{C_{1}}\left\|u_{0}\right\|_{2}^{2-p^{+}}\right), C_{1}, b_{0}$ are two positive constants.

Proof In Definition 2.1, we choose $u$ as a test-function to show

$$
\left.\frac{1}{2} \int_{\Omega} u^{2} d x\right|_{t_{1}} ^{t_{2}}+\int_{t_{1}}^{t_{2}} \int_{\Omega}|\nabla u|^{p(x, t)} d x d t+b_{0} \int_{t_{1}}^{t_{2}} \int_{\Omega} u^{2} d x d t=0
$$

Applying the conditions $\int_{t_{1}}^{t_{2}} \int_{\Omega}|\nabla u|^{p(x, t)} d x d t \leq \frac{1}{2}\left\|u_{0}\right\|_{2}^{2}$, the inequalities $\min \left\{\|u\|_{p(\cdot)}^{p^{-}}\right.$, $\left.\|u\|_{p(\cdot)}^{p^{+}}\right\} \leq \int_{t_{1}}^{t_{2}} \int_{\Omega}|\nabla u|^{p(x, t)} d x d t \leq \max \left\{\|u\|_{p(\cdot)}^{p^{-}},\|u\|_{p(\cdot)}^{p^{+}}\right\}$and the imbedding theorem $W_{0}^{1, p(x, t)}(\Omega) \hookrightarrow W_{0}^{1, p^{-}}(\Omega) \hookrightarrow L^{2}(\Omega)$, we have

$$
\begin{aligned}
\int_{t_{1}}^{t_{2}} \int_{\Omega}|\nabla u|^{p(x, t)} d x d t & \geq \min \left\{\|u\|_{p(\cdot)}^{p^{-}},\|u\|_{p(\cdot)}^{p^{+}}\right\} \\
& \geq C\left(p^{+}, p^{-}\right) \min \left\{1,\left\|u_{0}\right\|_{2}^{p^{-}-p^{+}}\right\}\|\nabla u\|_{p(\cdot)}^{p^{+}} \\
& \geq C\left(p^{+}, p^{-},\left\|u_{0}\right\|_{2}\right)\|\nabla u\|_{p^{-}}^{p^{+}} \geq C\left(p^{+}, p^{-},\left\|u_{0}\right\|_{2}\right)\|u\|_{2}^{p^{+}} .
\end{aligned}
$$


By (3.1), (3.2), we have

$$
\left.\frac{1}{2} \int_{\Omega} u^{2} d x\right|_{t_{1}} ^{t_{2}}+\int_{t_{1}}^{t_{2}}\left(\int_{\Omega}|u|^{2} d x\right)^{\frac{p^{+}}{2}} d t+b_{0} \int_{t_{1}}^{t_{2}} \int_{\Omega} u^{2} d x d t \leq 0
$$

In (3.3), let $t_{1}=t, t_{2}=t+h(0<h<T-t)$, multiply (3.3) by $h$ and apply Lebesgue's dominated convergence theorem to show that as $h \rightarrow 0$,

$$
\begin{aligned}
& \frac{d}{d t}\left(\int_{\Omega} u^{2}(x, t) d x\right)+2 C_{1}\left(\int_{\Omega}|u(x, t)|^{2} d x\right)^{\frac{p^{+}}{2}}+2 b_{0} \int_{\Omega} u^{2}(x, t) d x \\
& \quad \leq 0, \quad \text { a.e. } t \in(0, T) .
\end{aligned}
$$

By Gronwall's inequality, we have

$$
\int_{\Omega} u^{2} d x \leq\left[\left(\left\|u_{0}\right\|_{2}^{2-p^{+}}+\frac{b_{0}}{C_{1}}\right) e^{\left(\frac{p^{+}-2}{2}\right) b_{0} t}-\frac{b_{0}}{C_{1}}\right]^{\frac{2}{2-p^{+}}} .
$$

Theorem 3.2 Suppose that $b(x, t) \equiv 0,1<p^{-}<\frac{2 N}{N+2}, 1<p^{+}<\frac{N p^{-}}{N-p^{-}}$, then any bounded nonnegative solution of problem (1.1) vanishes in finite time for any nonnegative initial data $0 \not \equiv u_{0} \in L^{\infty}(\Omega) \cap W^{1, p(x)}(\Omega)$ and satisfies the following estimate:

$$
\begin{aligned}
& \|u\|_{r}^{r} \leq\left[\left(\left\|u_{0}\right\|_{r}^{r-\frac{r p^{+}\left(N-p^{-}\right)}{N p^{-}}}+\frac{C_{2}}{b_{0}}\right) e^{\left(\frac{p^{+}\left(N-p^{-}\right)}{N p^{-}}-1\right) b_{0} t}-\frac{C_{2}}{b_{0}}\right]^{\frac{N p^{-}}{N p^{-}-p^{+}\left(N-p^{-}\right)}}, \quad 0<t<T_{2}^{*} ; \\
& \|u\|_{r}=0, \quad t \in\left[T_{2}^{*},+\infty\right),
\end{aligned}
$$

where $r=\frac{N\left(2-p^{-}\right)}{p^{-}}, T_{2}^{*}=\frac{N p^{-}}{b_{0}\left(N p^{-}-p^{+}\left(N-p^{-}\right)\right)} \ln \left(1+\frac{b_{0}}{C_{2}}\left\|u_{0}\right\|_{r}^{r-\frac{r p^{+}\left(N-p^{-}\right)}{N p^{-}}}\right), C_{2}, b_{0}$ are two positive constants.

Proof In Definition 2.1, we choose $u^{\alpha}\left(\alpha=\frac{2 N-p^{-}(N+1)}{p^{-}}>1\right)$ as a test-function to show

$$
\begin{aligned}
& \left.\frac{1}{r} \int_{\Omega} u^{r} d x\right|_{t_{1}} ^{t_{2}}+C\left(\alpha,\left\|u_{0}\right\|_{\infty}, N, p^{ \pm}\right) \int_{t_{1}}^{t_{2}} \int_{\Omega}\left|\nabla u^{\beta}\right|^{p(x, t)} d x d t \\
& \quad+b_{0} \int_{t_{1}}^{t_{2}} \int_{\Omega} u^{r} d x d t \leq 0,
\end{aligned}
$$

where $r=\frac{N\left(2-p^{-}\right)}{p^{-}}>2, \beta=\frac{\left(2-p^{-}\right)\left(N-p^{-}\right)}{p^{-} p^{-}}>1$. The conditions $\int_{t_{1}}^{t_{2}} \int_{\Omega}|\nabla u|^{p(x, t)} d x d t \leq \frac{1}{2}\left\|u_{0}\right\|_{2}^{2}$, $\|u\|_{\infty, Q_{T}} \leq\left\|u_{0}\right\|_{\infty, \Omega}$, the inequalities $\min \left\{\|u\|_{p(\cdot)}^{p^{-}},\|u\|_{p(\cdot)}^{p^{+}}\right\} \leq \int_{t_{1}}^{t_{2}} \int_{\Omega}|\nabla u|^{p(x, t)} d x d t$ and the imbedding theorem $W_{0}^{1, p(x, t)}(\Omega) \hookrightarrow W_{0}^{1, p^{-}}(\Omega) \hookrightarrow L^{\frac{N p^{-}}{N-p^{-}}}(\Omega)$ show that the following inequalities hold:

$$
\begin{aligned}
\int_{t_{1}}^{t_{2}} \int_{\Omega}\left|\nabla u^{\beta}\right|^{p(x, t)} d x d t & \geq \min \left\{\|u\|_{p(\cdot)}^{p^{-}},\left\|u^{\beta}\right\|_{p(\cdot)}^{p^{+}}\right\} \\
& \geq C\left(p^{+}, p^{-}\right) \min \left\{1,\left\|u_{0}\right\|_{2}^{p^{-}-p^{+}}\right\}\|\nabla u\|_{p(\cdot)}^{p^{+}}
\end{aligned}
$$




$$
\begin{aligned}
& \geq C\left(p^{+}, p^{-},\left\|u_{0}\right\|_{2}\right)\left\|\nabla u^{\beta}\right\|_{p^{-}}^{p^{+}} \\
& \geq C\left(p^{+}, p^{-},\left\|u_{0}\right\|_{2}\right)\left\|u^{\beta}\right\|_{\frac{N^{+}}{N-p^{-}}}^{p^{+}}
\end{aligned}
$$

A similar argument as above gives that there exists a $T_{2}^{\prime \prime}>0$ such that $\|u\|_{r}$ satisfies that

$$
\begin{aligned}
& \|u\|_{r}^{r} \leq\left[\left(\left\|u_{0}\right\|_{r}^{r-\frac{r p^{+}\left(N-p^{-}\right)}{N p^{-}}}+\frac{C_{2}}{b_{0}}\right) e^{\left(\frac{p^{+}\left(N-p^{-}\right)}{N p^{-}}-1\right) b_{0} t}-\frac{C_{2}}{b_{0}}\right]^{\frac{N p^{-}}{N p^{-}-p^{+}\left(N-p^{-}\right)}}, \quad 0<t<T_{2}^{*} ; \\
& \|u\|_{r}=0, \quad t \in\left[T_{2}^{*},+\infty\right) .
\end{aligned}
$$

Remark 3.1 In the case when $1<p^{-}<\frac{2 N}{N+2}, 2>p^{+}>\frac{N p^{-}}{N-p^{-}}$, it is not clear whether any bounded nonnegative solution of problem (1.1) vanishes in finite time.

\section{Asymptotic behavior of weak solutions}

In this section, we study the asymptotic properties of the weak solution to problem (1.1). Namely, we study the long time asymptotic behavior of the solution, our main result is as follows.

Theorem 4.1 Suppose that $b(x, t) \equiv 0,2 \leq p^{-}<p^{+}$. If the following condition is satisfied

$\left(\mathrm{H}_{4}\right)$ there exists a positive continuous function $g(t)$ such that the following inequality holds:

$$
\int_{\Omega}|\nabla u(x, t)|^{p(x, t)} d x \leq M\left(u_{0}\right) g(t) \quad \text { for } t>0
$$

where $g(t)$ satisfies $\int_{0}^{+\infty} g^{\frac{2\left(p^{-}-p^{+}\right)}{p^{+} p^{-}}}(t) d t=+\infty$.

Then, for all $0<\sigma^{-}$, the solution to problem (1.1) satisfies

$$
\lim _{t \rightarrow+\infty}\|u(x, t)\|_{L^{2}(\Omega)}=0
$$

Proof Step 1. Let

$$
F(u)=\frac{1}{2} \int_{\Omega}|u|^{2} d x,
$$

then it is easy to prove that $F(u)$ is a convex functional on $L^{2}(\Omega)$.

For any $\tau \in(0, T)$ and $h>0$, by $\frac{\delta F(u)}{\delta u}=u$ ( $\delta$ representing Gâteaux differential) and the convexity of $F(u)$, we have

$$
F(u(\tau+h))-F(u(\tau)) \geq \int_{\Omega}(u(x, \tau+h)-u(x, \tau)) u(x, \tau) d x
$$

for any fixed $t_{1}, t_{2} \in[0, T], t_{1}<t_{2}$. Integrating inequality (4.1) with respect to $\tau$ over $\left(t_{1}, t_{2}\right)$, we have

$$
\begin{gathered}
\int_{t_{1}}^{t_{2}} F(u(\tau+h)) d \tau-\int_{t_{1}}^{t_{2}} F(u(\tau)) d \tau \\
=\int_{t_{1}+h}^{t_{2}+h} F(u(\tau)) d \tau-\int_{t_{1}}^{t_{2}} F(u(\tau)) d \tau
\end{gathered}
$$




$$
\begin{aligned}
& =\int_{t_{2}}^{t_{2}+h} F(u(\tau)) d \tau-\int_{t_{1}}^{t_{1}+h} F(u(\tau)) d \tau \\
& \geq \int_{t_{1}}^{t_{2}} \int_{\Omega}(u(x, \tau+h)-u(x, \tau)) u(x, \tau) d x d \tau .
\end{aligned}
$$

Multiplying both sides of (4.2) by $\frac{1}{h}$, and letting $h \rightarrow 0^{+}$, we obtain

$$
F\left(u\left(t_{2}\right)\right)-F\left(u\left(t_{1}\right)\right) \geq \int_{t_{1}}^{t_{2}} \int_{\Omega} \frac{\partial u}{\partial t} u d x d \tau .
$$

Similarly, we have

$$
F(u(\tau))-F(u(\tau-h)) \leq \int_{\Omega}(u(x, \tau)-u(x, \tau-h)) u(x, \tau) d x .
$$

Thus,

$$
F\left(u\left(t_{2}\right)\right)-F\left(u\left(t_{1}\right)\right) \leq \int_{t_{1}}^{t_{2}} \int_{\Omega} \frac{\partial u}{\partial t} u d x d \tau
$$

and hence

$$
F\left(u\left(t_{2}\right)\right)-F\left(u\left(t_{1}\right)\right)=\int_{t_{1}}^{t_{2}} \int_{\Omega} \frac{\partial u}{\partial t} u d x d \tau
$$

Choosing $t_{1}=0, t_{1}=t$, we get from the definition of solutions that

$$
F(u(t))-F(u(0))=-\int_{0}^{t} \int_{\Omega}\left(|u|^{\sigma}+d_{0}\right)|\nabla u|^{p(x)} d x d \tau-b_{0} \int_{0}^{t} \int_{\Omega}|u|^{2} d x d \tau .
$$

Step 2. We prove $u(x, t) \in C\left(0, T ; L^{2}(\Omega)\right)$. According to Theorem 2.1 and $\left(\mathrm{H}_{4}\right)$, we have the following conclusions:

$$
\left\{\begin{array}{l}
u_{\varepsilon} \in W\left(Q_{T}\right) \subseteq L^{\infty}\left(0, T ; W_{0}^{1, p^{-}}(\Omega)\right), \\
u_{\varepsilon t} \in W^{\prime}\left(Q_{T}\right) \subseteq L^{p^{+}} \\
\left.W_{0}^{1, p^{-}}(\Omega) \stackrel{\text { compact }}{\hookrightarrow} L^{2}(\Omega) \hookrightarrow V_{+}^{\prime}(\Omega)\right),
\end{array}\right.
$$

Applying Theorem 5 in [12], we get that the set $\left\{u_{\varepsilon}(x, t)\right\}$ is relatively compact in $C\left(0, T ; L^{2}(\Omega)\right)$, so $u(x, t) \in C\left(0, T ; L^{2}(\Omega)\right)$.

Step 3. Let $G(t)=\frac{1}{2} \int_{\Omega}|u(x, t)|^{2} d x$. Noting that $u(x, t) \in C\left(0, T ; L^{2}(\Omega)\right)$, then it is easy to prove that $G(t)$ is continuous in $(0, T)$, so we have $G^{\prime}(t)=-\int_{\Omega}\left(|u|^{\sigma}+d_{0}\right)|\nabla u|^{p(x)} d x-$ $b_{0} \int_{\Omega}|u|^{2} d x d \tau \leq 0$.

By the imbedding $W^{1, p(x)}(\Omega) \hookrightarrow W^{1, p^{-}}(\Omega) \hookrightarrow L^{2}(\Omega)$, Lemmas (2.1)-(2.3) in [5] and $\left(\mathrm{H}_{4}\right)$, we have

$$
\begin{aligned}
\int_{\Omega}|u|^{2} d x & \leq C\|u\|_{1, p(x)}^{2} \leq C\|\nabla u\|_{p(x)}^{2} \\
& \leq C\left(|\Omega|, p^{ \pm}\right) \max \left\{\left(\int_{\Omega}|\nabla u|^{p(x)} d x\right)^{\frac{2}{p^{-}}},\left(\int_{\Omega}|\nabla u|^{p(x)} d x\right)^{\frac{2}{p^{+}}}\right\}
\end{aligned}
$$




$$
\begin{aligned}
& \leq C\left(|\Omega|, p^{ \pm}, M\left(u_{0}\right), d_{0}\right) \max \left\{g^{\frac{2\left(p^{+}-p^{-}\right)}{p^{+} p^{-}}}(t), 1\right\}\left(\int_{\Omega}|\nabla u|^{p(x)} d x\right)^{\frac{2}{p^{+}}} \\
& \leq C\left(|\Omega|, p^{ \pm}, M\left(u_{0}\right), d_{0}\right) \max \left\{g^{\frac{2\left(p^{+}-p^{-}\right)}{p^{+} p^{-}}}(t), 1\right\}\left(\int_{\Omega} \frac{|u|^{\sigma}+d_{0}}{d_{0}}|\nabla u|^{p(x)} d x\right)^{\frac{2}{p^{+}}} \\
& \leq C\left(|\Omega|, p^{ \pm}, M\left(u_{0}\right), d_{0}, \alpha\right) \max \left\{g^{\frac{2\left(p^{+}-p^{-}\right)}{p^{+} p^{-}}}(t), 1\right\}\left|G^{\prime}(t)\right|^{\frac{2}{p^{+}}}
\end{aligned}
$$

that is, $G(t) \leq C \max \left\{g^{\frac{2\left(p^{+}-p^{-}\right)}{p^{+} p^{-}}}(t), 1\right\}\left|G^{\prime}(t)\right|^{\frac{2}{p^{+}}}$.

Noting that $G(t) \geq 0, G^{\prime}(t) \leq 0$, we have $G^{\prime}(t) \leq-\frac{C G^{\frac{p^{+}}{2}}(t)}{1+g \frac{2\left(p^{+}-p^{-}\right)}{p^{+} p^{-}}}(t)$, and hence

$$
\int_{\Omega}|u(x, T)|^{2} d x \leq \frac{1}{\left[C_{1} \int_{0}^{T}\left(1+g^{\frac{2\left(p^{+}-p^{-}\right)}{p^{+} p^{-}}}(t)\right)^{-1} d t+C_{2}\right]^{\delta}}, \quad \delta=\frac{2}{p^{+}-2}, \quad C_{i}>0, i=1,2 .
$$

This completes the proof of Theorem 4.1.

\section{Competing interests}

The authors declare that they have no competing interests.

\section{Authors' contributions}

Both authors collaborated in all the steps concerning the research and achievements presented in the final manuscript.

\section{Acknowledgements}

The work was supported by the Natural Science Foundation of China (11271154) and by the 985 program of Jilin University. We are very grateful to the anonymous referees for their valuable suggestions that improved the article.

Received: 11 March 2013 Accepted: 26 June 2013 Published: 10 July 2013

\section{References}

1. Wei, DM: Existence, uniqueness and numerical analysis of solutions of a quasilinear parabolic problem. SIAM J. Numer. Anal. 29(2), 484-497 (1992)

2. Dibenedetto, E: Degenerate Parabolic Equations. Springer, New York (1993)

3. Zhao, JN: On the Cauchy problem and initial traces for the evolution $p$-Laplacian equations with strongly nonlinear sources. J. Differ. Equ. 121, 329-383 (1995)

4. Ruzicka, M: Electrorheological Fluids: Modelling and Mathematical Theory. Lecture Notes in Math., vol. 1748. Springer, Berlin (2000)

5. Antontsev, SN, Shmarev, SI: Anisotropic parabolic equations with variable nonlinearity. Publ. Math. 53, 355-399 (2009)

6. Chen, Y, Levine, S, Rao, M: Variable exponent, linear growth functionals in image restoration. SIAM J. Appl. Math. 66, 1383-1406 (2006)

7. Acerbi, E, Mingione, G, Seregin, GA: Regularity results for parabolic systems related to a class of non newtonian fluids. Ann. Inst. Henri Poincaré, Anal. Non Linéaire, 21, 25-60 (2004)

8. Acerbi, E, Mingione, G: Regularity results for a class of functionals with nonstandard growth. Arch. Ration. Mech. Anal. 156(1), 121-140(2001)

9. Diening, L, Harjulehto, P, Hästö, P, Rûžička, M: Lebesgue and Sobolev Spaces with Variable Exponents. Lecture Notes in Mathematics, vol. 2017. Springer, Heidelberg (2011)

10. Guo, B, Gao, WJ: Study of weak solutions for parabolic equations with nonstandard growth conditions. J. Math. Anal. Appl. 374(2), 374-384 (2011)

11. Guo, B, Gao, WJ: Existence and asymptotic behavior of solutions for nonlinear parabolic equations with variable exponent of nonlinearity. Acta Math. Sci. 32(3), 1053-1062 (2012)

12. Simon, J: Compact sets in the space $L^{P}(0, T ; B)$. Ann. Math. Pures Appl. 4(146), 65-96 (1987)

doi:10.1186/1687-2770-2013-164

Cite this article as: Gao and Gao: Extinction and asymptotic behavior of solutions for nonlinear parabolic equations with variable exponent of nonlinearity. Boundary Value Problems 2013 2013:164. 